\title{
RTM results for Predicting Translation Performance
}

\author{
Ergun Biçici \\ ergun.bicici@boun.edu.tr \\ Department of Computer Engineering, Boğaziçi University \\ orcid.org/0000-0002-2293-2031 \\ bicici.github.com
}

\begin{abstract}
With improved prediction combination using weights based on their training performance and stacking and multilayer perceptrons to build deeper prediction models, RTMs become the 3rd system in general at the sentence-level prediction of translation scores and achieve the lowest RMSE in English to German NMT QET results. For the document-level task, we compare document-level RTM models with sentence-level RTM models obtained with the concatenation of document sentences and obtain similar results.
\end{abstract}

\section{Introduction}

Quality estimation task in WMT18 (Specia et al., 2018) (QET18) address machine translation performance prediction (MTPP), where translation quality is predicted without using reference translations, at the sentence- (Task 1), word- (Task 2), phrase-level (Task 3), and document-levels (Task 4). The tasks contain subtasks involving EnglishGerman phrase-based machine translation (SMT) and neural network-based SMT (NMT), GermanEnglish SMT, English-Latvian SMT and NMT, English-Czech SMT, and English-French SMT. Task 1 is about predicting HTER (human-targeted translation edit rate) scores (Snover et al., 2006), Task 2 is about binary classification of words, Task 3 is about binary classification of phrases, and Task 4 is about predicting multi-dimensional quality metrics (MQM) (Lommel, 2015).

We use referential translation machine (RTM) (Biçici, 2017) models for building our prediction models. RTMs predict data translation between the instances in the training set and the test set using interpretants, data close to the task instances. Interpretants provide context for the prediction task and are used during the derivation of the features measuring the closeness of the

\begin{tabular}{l|rr|rr} 
& & \multicolumn{2}{|c}{ RTM interpretants } \\
Task & Train & Test & Training & LM \\
\hline Task 1 (en-cs, SMT) & 41254 & 1000 & & \\
Task 1 (en-de, SMT) & 27273 & 1000 & & \\
Task 1 (en-de, NMT) & 14442 & 1000 & & \\
Task 1 (de-en, SMT) & 26963 & 1000 & & \\
Task 1 (en-lv, SMT) & 12251 & 1000 & $0.225 \mathrm{M}$ & $5 \mathrm{M}$ \\
Task 1 (en-lv, NMT) & 13936 & 1000 & & \\
Task 1 (en-lv, NMT) & 13936 & 1000 & \\
Task 3 (de-en, NMT) & 6021 & 543 & \\
Task 4 (en-fr, NMT) & 1200 & 269 & \\
\hline
\end{tabular}

Table 1: Number of instances and interpretants used.

test sentences to the training data, the difficulty of translating them, and to identify translation acts between any two data sets for building prediction models. With the enlarging parallel and monolingual corpora made available by WMT, the capability of the interpretant datasets selected by RTM models to provide context for the training and test sets improve.

Figure 1 depicts RTMs and explains the model building process. RTMs use parfda (Bicici, 2018) for instance selection and machine translation performance prediction system (MTPPS) (Biçici and Way, 2015) for generating features. The total number of features vary depending on the order of $n$-grams used (e.g. a log of probability score from the language model for each $n$-gram is used).

We use ridge regression, kernel ridge regression, k-nearest neighors, support vector regression, AdaBoost (Freund and Schapire, 1997), gradient tree boosting, extremely randomized trees (Geurts et al., 2006), and multi-layer perceptron (Bishop, 2006) as learning models in combination with feature selection (FS) (Guyon et al., 2002) and partial least squares (PLS) (Wold et al., 1984) where most of these models can be found in scikit-learn. ${ }^{1}$ Evaluation metrics listed

\footnotetext{
${ }^{1}$ http://scikit-learn.org/
} 


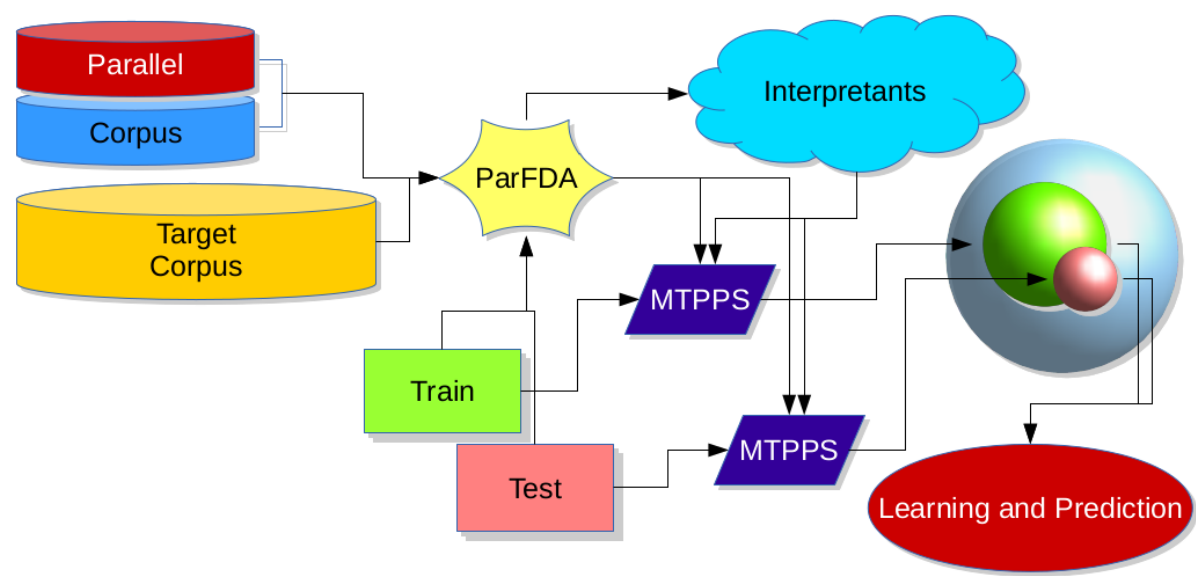

Figure 1: RTM depiction: ParFDA selects interpretants close to the training and test data using parallel corpus in bilingual settings and monolingual corpus in the target language or just the monolingual target corpus in monolingual settings; an MTPPS use interpretants and training data to generate training features and another use interpretants and test data to generate test features in the same feature space; learning and prediction takes place taking these features as input.

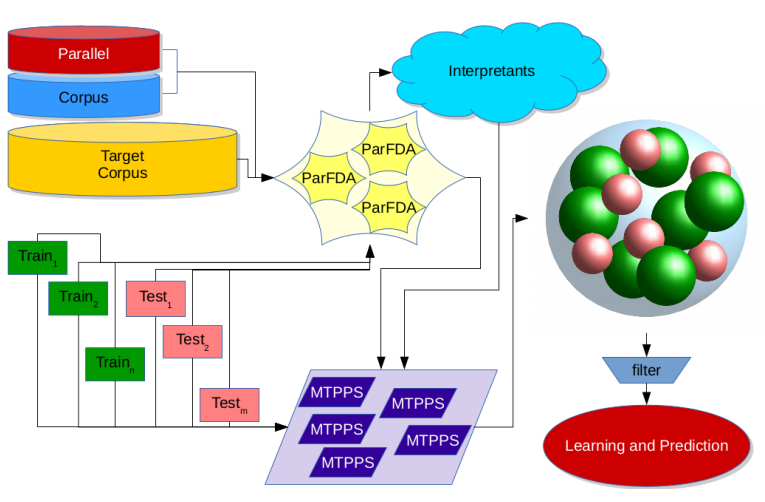

Figure 2: Document-level RTM model with separate MTPPS run for each training and test document to obtain corresponding feature representations, which are filtered and processed before learning and prediction.

are Pearson's correlation $(r)$, mean absolute error (MAE), and root mean squared error (RMSE).

We use Global Linear Models (GLM) (Collins, 2002) with dynamic learning (GLMd) (Biçici, 2017) for word- and phrase-level translation performance prediction. GLMd uses weights in a range $[a, b]$ to update the learning rate dynamically according to the error rate.

\section{Mixture of Experts Models}

We use prediction averaging (Biçici, 2017) to obtain a combined prediction from various prediction outputs better than the components, where the performance on the training set is used to obtain weighted average of the top $k$ predictions, $\hat{y}$ with evaluation metrics indexed by $j \in J$ and weights

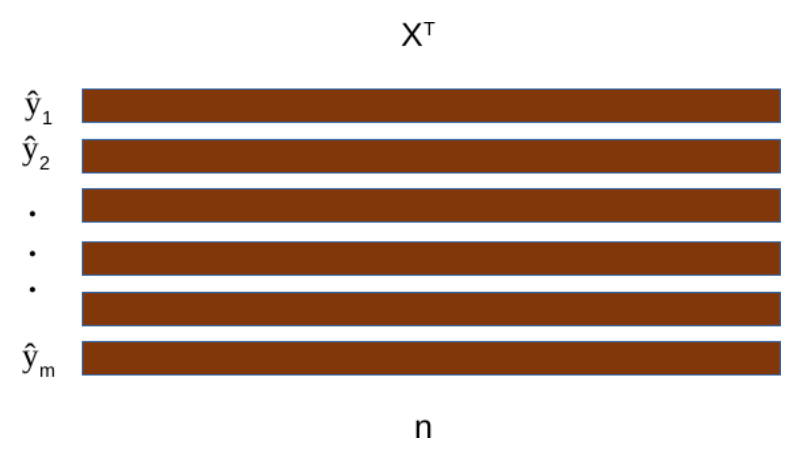

Figure 3: Stacking training data, $\mathrm{X}$, from $m$ predictors.

with $w$ :

$$
\begin{aligned}
& w_{j, i}= \begin{cases}\frac{1}{\mathrm{eval}_{j, i}} & \text { if } j \text { is minimized } \\
\mathrm{eval}_{j, i} & \text { if } j \text { is maximized }\end{cases} \\
& \hat{\boldsymbol{y}}_{\mu_{k}}=\frac{1}{k} \sum_{i=1}^{k} \hat{\boldsymbol{y}}_{i} \\
& \hat{\boldsymbol{y}}_{j, w_{k}^{j}}=\frac{1}{\sum_{i=1}^{k} w_{j, i}} \sum_{i=1}^{k} w_{j, i} \hat{\boldsymbol{y}}_{i} \\
& \hat{\boldsymbol{y}}_{k}=\frac{1}{|J|} \sum_{j \in J} \hat{\boldsymbol{y}}_{j, w_{k}^{j}}
\end{aligned}
$$

where weights are inverted to decrease error. We only use the MIX prediction if we obtain better results on the training set. We select the best model using $r$ and mix the results using $r$, RAE, MRAER, and MAER. The set of evaluation metrics used for mixing also affects the results. Since we try to obtain results with relative evaluation metric scores less than 1 , we filter out those results with higher than 1 relative evaluation metric scores.

In our experiments, we found that assuming independent predictions and using $p_{i} /\left(1-p_{i}\right)$ for 


\begin{tabular}{lll|lllll} 
Task 4 & model & setting & $r_{P}$ & MAE & RAE & MAER & MRAER \\
\hline en-fr SMT & doc & stack & -0.1725 & 43.0687 & 0.9857 & 0.8123 & 0.805 \\
en-fr SMT & doc & mix & -0.1812 & 43.5726 & 0.9973 & 0.8347 & 0.8256 \\
en-fr SMT & doc & FS RR & -0.177 & 44.058 & 1.0084 & 0.8509 & 0.8413 \\
en-fr SMT & sent & stack & 0.2109 & 42.5196 & 0.9732 & 0.8464 & 0.8372 \\
en-fr SMT & sent & mix & -0.2299 & 43.2402 & 0.9897 & 0.8197 & 0.8116 \\
en-fr SMT & sent & FS KR & -0.1844 & 43.2891 & 0.9908 & 0.8255 & 0.8167 \\
\hline
\end{tabular}

Table 2: Training results on Task 4 with stacking and prediction averaging. FS RR is the top single model for doc and FS KR for sent where RR is ridge regression and KR is kernel ridge regression.

\begin{tabular}{l|lllll} 
Task 1 & $r_{P}$ & MAE & RAE & MAER & MRAER \\
\hline en-de SMT & 0.4336 & 0.1365 & 0.8654 & 0.7951 & 0.798 \\
en-de NMT & 0.459 & 0.1138 & 0.8282 & 0.84 & 0.7771 \\
de-en SMT & 0.5634 & 0.1364 & 0.7953 & 0.7637 & 0.7573 \\
en-cs SMT & 0.5381 & 0.151 & 0.8109 & 0.7423 & 0.7679 \\
en-lv SMT & 0.3805 & 0.1362 & 0.9055 & 0.8755 & 0.8041 \\
en-lv NMT & 0.5714 & 0.1466 & 0.7971 & 0.753 & 0.7595 \\
\hline
\end{tabular}

Table 3: Training results on Task 1 with prediction averaging.

weights where $p_{i}$ represents the accuracy of the independent classifier $i$ in a weighted majority ensemble (Kuncheva and Rodríguez, 2014) obtained slightly better results (Equation (2)).

$$
w_{j, i}=\frac{w_{j, i}}{1-w_{j, i}}
$$

We also use stacking to build higher level models using predictions from base prediction models where they can also use the probability associated with the predictions (Ting and Witten, 1999). The stacking models use the predictions from predictors as features and build second level predictors (Figure 3).

\section{Document-level MTPP Model Comparisons}

We evaluate the effect of two different RTM data modeling techniques for the document-level task. Our first approach involves running separate MTPPS instances for each training (green in Figure 2) or test (salmon colored) document to obtain specific features for each document. Then, only the document-level features and the min, max, and average of the sentence-level features are used to obtain an RTM representation vector instance from each document. Our second approach concatenates the sentences from each document to obtain a single sentence representing each and runs an RTM model. Features from word alignment are included in both and they share the interpretants. The first approach use 1359 features and the second use 383 features.

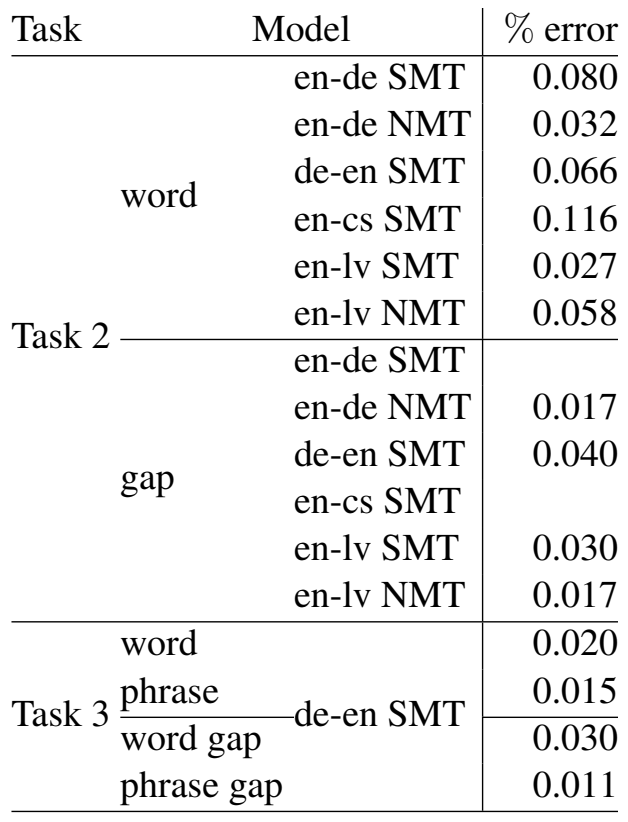

Table 4: RTM Task 2 training error for some of the models where GLMd is parallelized over splits. All GLMd models use $[0.5,2]$ as weights. \% error are twice the overall error found based on all tags $(2 \mathrm{~N}+1)$.

Training results are in Table 2 where we compare them and the first approach is denoted as doc and the second as sent. The first approach obtained the top results in QET16 (Bicici, 2016). doc obtains better MAER (mean absolute error relative) and MRAER (mean relative absolute error relative) (Biçici and Way, 2015). We obtain 3rd best RMSE while we note that both MAE and RMSE results are close to each other in all four submissions on the test set.

\section{Results}

Table 1 lists the number of sentences in the training and test sets for each task and the number of instances used as interpretants in the RTM models ( $\mathrm{M}$ for million). We tokenize and truecase all of the corpora using Moses' (Koehn et al., 


\begin{tabular}{llll|lll}
\multicolumn{1}{c}{ Task 4 } & model & setting & $r_{P}$ & MAE & RMSE \\
\hline top & & & & 0.5337 & 56.2264 & 85.2319 \\
& en-fr SMT & doc & stack & $0.0580(4)$ & $58.5680(4)$ & $87.8321(4)$ \\
\multirow{2}{*}{ RTM } & en-fr SMT & doc & mix & $-0.1210(4)$ & $57.5613(4)$ & $86.2219(4)$ \\
& en-fr SMT & sent & stack & $0.0183(4)$ & $57.6245(4)$ & $86.4831(4)$ \\
& en-fr SMT & sent & mix & $-0.0812(4)$ & $57.7922(4)$ & $86.8650(4)$ \\
\hline
\end{tabular}

Table 5: Task 4 test RTM results and the top result in the task.

\begin{tabular}{|c|c|c|c|c|}
\hline Task 1 & $r_{P}$ & $r_{S}$ & MAE & RMSE \\
\hline SMT top & 0.7397 & 0.7543 & 0.0937 & 0.1362 \\
\hline RTM & $0.4166(6)$ & $0.4254(4)$ & $0.1353(5)$ & $0.1731(6)$ \\
\hline $\mathrm{TT}^{\text {top }}$ & 0.5129 & 0.6052 & 0.1114 & 0.1719 \\
\hline RTM & $0.4704(3)$ & $0.5461(3)$ & $0.1192(3)$ & 0.1727 (1) \\
\hline de-en SMT top & 0.7667 & 0.7318 & 0.0945 & 0.1315 \\
\hline RTM & $0.5772(6)$ & $0.5167(5)$ & $0.1311(6)$ & $0.1679(4)$ \\
\hline en-cs SMT top & 0.6918 & 0.7105 & 0.1223 & 0.1693 \\
\hline RTM & 0.5295 (3) & $0.5348(3)$ & 0.1519 (3) & $0.1952(3)$ \\
\hline -ly SMT top & 0.6188 & 0.5766 & 0.1202 & 0.1602 \\
\hline RTM & $0.3521(8)$ & $0.2861(7)$ & $0.1430(4)$ & $0.1869(3)$ \\
\hline en-ly NMT top & 0.6819 & 0.6665 & 0.1308 & 0.1747 \\
\hline RTM & $0.5487(4)$ & 0.5017 (4) & $0.1540(3)$ & $0.2006(3)$ \\
\hline
\end{tabular}

Table 6: Test results of RTM in Task 1 where numbers in parentheses show the rank and corresponding top results. RTM achieves the lowest RMSE in en-de NMT and becomes the 3rd system in general. $r_{P}$ is Pearson's correlation and $r_{S}$ is Spearman's correlation.

2007) processing tools. ${ }^{2}$ LMs are built using ken Im (Heafield et al., 2013). The comparison of results on the training set are in Table 3 for Task 1 and in Table 2 for Task 4.

The results on the test set (Tables 5 and 6) shows that RTM can become the 1st in en-de NMT and 3 rd in general. Test results are taken from the competition's result submission websites at:

- sentence level https://competitions. codalab.org/competitions/19316

- word level https://competitions. codalab.org/competitions/19306

- phrase level https://competitions. codalab.org/competitions/19308

- document level https://competitions. codalab.org/competitions/19309

The references for the test sets are not released yet.

For Task 2 and Task 3, we model words or phrases and gaps separately and then combine their results. The error $\%$ on the training sets are in Table 4 and the results on the test set are in Table 7 .

\footnotetext{
${ }^{2}$ https://github.com/moses-smt/ mosesdecoder/tree/master/scripts
}

\begin{tabular}{|c|c|c|c|c|}
\hline & sk & $F_{1} \mathrm{BAD}$ & $F_{1} \mathrm{OK}$ & $\mathrm{w} F_{1}$ \\
\hline \multirow{3}{*}{$\begin{array}{l}\text { en-de } \\
\text { SMT }\end{array}$} & word & $3300(7)$ & $0.8813(3)$ & \\
\hline & & 2547 (3) & 0.9764 (1) & 0.2487 (3) \\
\hline & src & $1650(2)$ & 0.8591 (1) & $0.1418(2)$ \\
\hline \multirow{3}{*}{$\begin{array}{l}\text { en-de } \\
\text { NMT }\end{array}$} & word & 0927 (6) & 0.9235 (1) & $0.0856(6)$ \\
\hline & & & $0.9878(1)$ & $0.1343(1)$ \\
\hline & src & & & 0.0 \\
\hline \multirow{3}{*}{$\begin{array}{l}\text { de-en } \\
\text { SMT }\end{array}$} & word & & & 0.3 \\
\hline & & & & \\
\hline & src & $0.1211(2)$ & $0.8946(1)$ & $0.1083(2)$ \\
\hline \multirow{3}{*}{$\begin{array}{l}\text { en-lv } \\
\text { SMT }\end{array}$} & word & 3) & 0.9044 (1) & 0.3329 (3 \\
\hline & gap & & & 0.1279 \\
\hline & src & & 0.8 & $0.1066(2)$ \\
\hline \multirow{3}{*}{$\begin{array}{l}\text { en-lv } \\
\text { NMT }\end{array}$} & word & & $0.8530(1)$ & $0.3651(3)$ \\
\hline & $\mathrm{a}$ & 3) & 0.9819 (1) & $0.0814(3$ \\
\hline & & & & 0.16 \\
\hline \multirow{3}{*}{$\begin{array}{l}\text { en-cs } \\
\text { SMT }\end{array}$} & word & & 0.8 & $0.4360(4$ \\
\hline & gap & & & 0.1039 \\
\hline & src & 2) & 0.7962 & $0.2571(2$ \\
\hline \multirow{6}{*}{\begin{tabular}{ll} 
& \\
\multirow{Z}{*}{} & $\mathrm{de}-\mathrm{en}$ \\
$\mathrm{H}$ & $\mathrm{SMT}$
\end{tabular}} & phras & ) & 0.9 & $0.2431(2)$ \\
\hline & gap & & & 0.0508 \\
\hline & $\mathrm{src}$ & $0.0956(1)$ & 0.8994 (1) & 0.0860 \\
\hline & $\overline{\text { word }}$ & $.1648(3)$ & $0.9004(2)$ & $0.1484(3)$ \\
\hline & & & & 0.0964 (2) \\
\hline & & & $0.8376(1)$ & $0.0815(2$ \\
\hline
\end{tabular}

Table 7: RTM Task 2 and Task 3 results on the test set. w $F_{1}$ is average weighted $F_{1}$ score ( $F_{1}$ multi).

\section{Conclusion}

Referential translation machines can achieve top performance in automatic, accurate, and language independent prediction of translation scores and achieve to become the 1st system according to RMSE for MTPP from English to German in QET18. RTMs pioneer a language independent approach and remove the need to access any task or domain specific information or resource.

\section{Acknowledgments}

The research reported here received financial support in part from the Scientific and Technological Research Council of Turkey (TÜBITAK) with- 
out contribution to the content nor responsibility thereof. We also thank the reviewers' comments and Fred Blain from The University of Sheffield.

\section{References}

Ergun Biçici. 2017. Predicting translation performance with referential translation machines. In Proc. of the Second Conference on Statistical Machine Translation (WMT17), pages 540-544, Copenhagen, Denmark. Association for Computational Linguistics.

Ergun Biçici and Andy Way. 2015. Referential translation machines for predicting semantic similarity. Language Resources and Evaluation, pages 1-27.

Ergun Bicici. 2016. Referential translation machines for predicting translation performance. In Proc. of the First Conference on Statistical Machine Translation (WMT16), pages 777-781, Berlin, Germany. Association for Computational Linguistics.

Ergun Bicici. 2018. Robust parfda statistical machine translation results. In Proc. of the Third Conference on Statistical Machine Translation (WMT18), Brussels, Belgium.

Christopher M. Bishop. 2006. Pattern Recognition and Machine Learning.

Michael Collins. 2002. Discriminative training methods for hidden markov models: theory and experiments with perceptron algorithms. In Proc. of the ACL-02 conference on Empirical methods in natural language processing - Volume 10, EMNLP '02, pages $1-8$, Stroudsburg, PA, USA.

Yoav Freund and Robert E Schapire. 1997. A decisiontheoretic generalization of on-line learning and an application to boosting. Journal of Computer and System Sciences, 55(1):119-139.

Pierre Geurts, Damien Ernst, and Louis Wehenkel. 2006. Extremely randomized trees. Machine Learning, 63(1):3-42.

Isabelle Guyon, Jason Weston, Stephen Barnhill, and Vladimir Vapnik. 2002. Gene selection for cancer classification using support vector machines. $\mathrm{Ma}$ chine Learning, 46(1-3):389-422.

Kenneth Heafield, Ivan Pouzyrevsky, Jonathan H. Clark, and Philipp Koehn. 2013. Scalable modified Kneser-Ney language model estimation. In Proc. of the 51 st Annual Meeting of the Association for Computational Linguistics, pages 690-696, Sofia, Bulgaria.

Philipp Koehn, Hieu Hoang, Alexandra Birch, Chris Callison-Burch, Marcello Federico, Nicola Bertoldi, Brooke Cowan, Wade Shen, Christine Moran, Richard Zens, Chris Dyer, Ondrej Bojar, Alexandra Constantin, and Evan Herbst. 2007. Moses: Open source toolkit for statistical machine translation. In
Proc. of the 45th Annual Meeting of the Association for Computational Linguistics Companion Volume Proc. of the Demo and Poster Sessions, pages 177180. Association for Computational Linguistics.

Ludmila I. Kuncheva and Juan J. Rodríguez. 2014. A weighted voting framework for classifiers ensembles. Knowledge and Information Systems, 38(2):259-275.

Arle Lommel. 2015. Multidimensional quality metrics (mqm) definition. URL http://www. qt21. eu/mqmdefinition/definition-2015-12-30. html.

Matthew Snover, Bonnie Dorr, Richard Schwartz, Linnea Micciulla, and John Makhoul. 2006. A Study of Translation Edit Rate with Targeted Human Annotation. In Proc. of Association for Machine Translation in the Americas,

Lucia Specia, Frédéric Blain, Varvara Logacheva, Ramón F. Astudillo, and André Martins. 2018. Findings of the wmt 2018 shared task on quality estimation. In Proceedings of the Third Conference on Machine Translation, Volume 2: Shared Task Papers, Brussels, Belgium. Association for Computational Linguistics.

Kai Ming Ting and Ian H. Witten. 1999. Issues in stacked generalization. Journal of Artificial Intelligence Research, 10:271-289.

S. Wold, A. Ruhe, H. Wold, and III Dunn, W. J. 1984. The collinearity problem in linear regression. the partial least squares (pls) approach to generalized inverses. SIAM Journal on Scientific and Statistical Computing, 5:735-743. 